\title{
PSICOLOGIA
}

Revista da Associação Portuguesa de Psicologia (Edição Online)

Volume XXXI, Número 2, 2017

\section{Número Temático: Estudos sobre Bullying em Portugal}

Eds. Ana Tomás de Almeida e Isabel Correia

Propriedade e Edição da Associação Portuguesa de Psicologia

ISSN: 2183-2471 


\section{Revista PSICOLOGIA}

Director:

António J. Santos, Departamento de Ciências Psicológicas do ISPA-Instituto Universitário

\section{Directores Associados}

Marco Pereira, Faculdade de Psicologia e de Ciências da Educação da Universidade de Coimbra

Pedro Dias, Faculdade de Educação e Psicologia da Universidade Católica Portuguesa

Adriana Sampaio, Escola de Psicologia da Universidade do Minho

David Rodrigues, Centro de Investigação e Intervenção Social do ISCTE-IUL

Secretariado Científico

Olívia Ribeiro, William James Center for Research do ISPA-Instituto Universitário

\section{Secretariado Técnico}

Margarida Carmona e Lima, Associação Portuguesa de Psicologia

\section{Conselho Editorial}

Alexandra Reis (Universidade do Algarve, Portugal)

António Rosado (FMH-Universidade de Lisboa, Portugal)

Cícero Roberto Pereira (Universidade Federal da Paraíba, Brasil)

Dominique Muller (Université de Grenoble Alpes, França)

Ewa Drozda-Senkowska (Université Paris Descartes, França)

Gerd Bohner (Fakultät für Psychologie und Sportwissenschaft,

Universität Bielefield, Alemanha)

Gün R. Semin (ISPA-Instituto Universitário, Portugal)

Isabel Correia (ISCTE-IUL, Portugal)

João Manuel de Oliveira (ISCTE-IUL, Portugal)

Jorge Vala (Instituto de Ciências Sociais, Universidade de

Lisboa, Portugal)

José Marques (FPCE-Universidade do Porto, Portugal)

Jose-Miguel Fernández-Dols (Universidad Autónoma de

Madrid, Espanha)

Liliana Sousa (Universidade de Aveiro, Portugal)

Luís Faísca (Universidade do Algarve, Portugal)

Luísa Lima (ISCTE-IUL, Portugal)

Manuela Calheiros (ISCTE-IUL, Portugal)

Marcus Lima (Universidade Federal do Sergipe, Brasil)

Margarida Garrido (ISCTE-IUL, Portugal)

Maria Benedicta Monteiro (ISCTE-IUL, Portugal)

Maria Filomena Gaspar (Universidade de Coimbra, Portugal)

Mário Boto Ferreira (FP-Universidade de Lisboa, Portugal)

Marlene Matos (Universidade do Minho, Portugal)

Miguel Gonçalves (Universidade do Minho, Portugal)

Patrícia Arriaga (ISCTE-IUL, Portugal)

Paulo Machado (Universidade do Minho, Portugal)

São Luís Castro (FPCE-Universidade do Porto, Portugal)

Silvia Koller (Universidade Federal do Rio Grande do Sul, Brasil)

Teresa Garcia-Marques (ISPA-Instituto Universitário, Portugal)

Themis Apostolidis (Université Aix-Marseille, França)

Theodore Alexopoulos (Université Paris Descartes, França)

Theodore E. A. Waters (New York University Abu Dhabi, Emirados Árabes Unidos)

A revista PSICOLOGIA é uma revista multilingue e encontra-se indexada nos PSYCHOLOGICAL ABSTRACTS / PSYARTICLES /

PSYCINFO / SCIELO / SCIELO Citation Index - Thomson Reuters / SCOPUS

Propriedade do Título, Edição, Concepção Gráfica e Composição: Associação Portuguesa de Psicologia (http://appsicologia.org) Capa: (c) Associação Portuguesa de Psicologia

Depósito Legal número: 129 801/98

Registo no Instituto de Comunicação Social número: 124021

ISSN: 2183-2471

Revisão de textos: Maria do Carmo Carvalho

Preço deste número: €15. Assinatura anual (dois números): Subscrição individual - €30, Estudantes - €20, Instituições - €40.

Pedidos de assinaturas ou compra de números avulso devem ser solicitados para revista@appsicologia.org, ou através do portal da revista em http://revista.appsicologia.org/index.php/rpsicologia/about/subscriptions

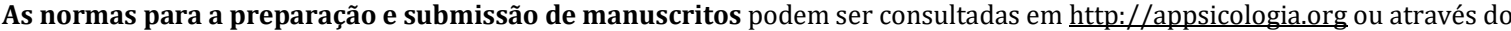
portal da revista em http://revista.appsicologia.org/index.php/rpsicologia/about/submissions\#onlineSubmissions

Sede da redacção: Associação Portuguesa de Psicologia, Edifício ISCTE-IUL, Sala 0w5, Av. das Forças Armadas, 1649-026 Lisboa, Portugal 


\section{Revista PSICOLOGIA}

Volume 31 (2), 2017

Índice

Secção Temática

Número Temático sobre Bullying em Portugal (pp. iv-xi)

Ana Tomás de Almeida e Isabel Correia

Comportamentos e motivos dos/as observadores/as de bullying: contributos para a sua avaliação (pp. 1-14)

Madalena Melo e Sónia Pereira

Having friends with gay friends? The role of extended contact, empathy and threat on assertive bystanders behavioral intentions (pp. 15-24)

Raquel António, Rita Guerra e Carla Moleiro

Mecanismos de proteção perante a vitimização por pares e a discriminação (pp. 25-46)

Daniela Fonseca Freitas, Susana Coimbra, Anne Marie Fontaine e Edna Maria Marturano

Popular justice on bullies: Attributions of responsibility, anger, and ineffective social control as fuel for non-normative collective action (pp. 47-56)

Miguel Campos, André G. Marques, Tiago Aguiar, Sónia G. Cardoso, Isabel R. Pinto e José M. Marques

Violência entre pares na adolescência: Um estudo com estudantes no início e no final do $3 .^{\circ}$ ciclo do ensino básico (pp. 57-68)

Ana Cristina Rosário, Adelinda Candeias e Madalena Melo

Bullying e agressão: Estudo dos preditores no contexto de programa de intervenção da violência escolar (pp. 69-88)

Patrícia Gouveia, Isabel Leal e Jorge Cardoso

\section{Secção Não-Temática}

Os artigos que compõem esta secção foram submetidos e aceites na vigência da Direcção anterior da revista PSICOLOGIA, sob a Coordenação do Doutor Mário B. Ferreira

Locus de controlo e adesão terapêutica em adultos emergentes e adultos com condições crónicas de saúde: 0 papel moderador do grupo etário (pp. 89-104)

Jéssica Reis, Raquel Pires e Neuza Silva

Impacto das missões internacionais nos jovens filhos de pais militares e a importância do apoio da comunidade escolar (pp. 105-120)

Rita Pinto, Rita Francisco e Renato Pessoa dos Santos

Psychometric properties of the Portuguese version of the Relational Interdependent Self Construal scale (pp. 121-136)

Gabriela Gonçalves, Cátia Sousa, Joana Santos, Alexandra Gomes, Andreia Santa-Rita, Sofia Hipólito, Catarina Rodrigues e Marta Reis

\section{Atas do IX Simpósio Nacional de Investigação em Psicologia}

Editorial (pp. xii-xiv)

Diniz Lopes e Margarida Garrido (Organizadores)

Atas do IX Simpósio Nacional de Investigação em Psicologia (pp. 137-328) 


\section{EDITORIAL}

\section{Número Temático sobre Bullying em Portugal}

Gostaríamos de expressar o nosso agradecimento à revista de Psicologia por acolher um número temático dedicado ao Bullying. Esta edição parece-nos particularmente relevante por vários motivos.

Primeiro, o tema continua a despertar a atenção da comunidade científica em Portugal. A produção de estudos sobre bullying que é possível rastrear nos repositórios nacionais de acesso aberto dá nota do aumento consistente de trabalhos académicos produzidos no âmbito de dissertações de mestrado e teses de doutoramento nas instituições nacionais de ensino superior públicas e privadas. Não obstante, e apesar do carácter incompleto e das descontinuidades que garantidamente existem neste tipo de registo, é possível afirmar que, na última década, o tema tem atraído um conjunto vasto de investigações relacionadas com agressores e vítimas que, sob o prisma da agressão ou pelo da vitimação, tem ajudado a dimensionar, caraterizar e ampliar os contornos do fenómeno da violência entre pares em contexto escolar, o que, em si mesmo, tem sido útil para monitorar a realidade nacional.

Segundo, ao convite lançado para este número temático da revista de Psicologia respondem maioritariamente alunos e docentes de programas de pós-graduação de universidades portuguesas, alguns destes integrando unidades de investigação das escolas respetivas. Apraz-nos registar a resposta desta comunidade de académicos e investigadores que submete os trabalhos para revisão pelos pares, procedendo de maneira a ajustar a produção académica às práticas de divulgação científica. É esta dinâmica e a consciencialização da importância em dar visibilidade à produção académica, contrariando a tendência de a rotular de 'cinzenta', que contribui para a melhoria da investigação que se produz e publica em Portugal. Nesse sentido, um número temático pode também apoiar a qualidade e a sustentabilidade das redes de investigadores.

Terceiro, a seleção de artigos que integra este número temático representa uma pequena amostra dos estudos sobre bullying que se realizam no contexto nacional e, ainda assim, como trataremos abaixo na brevíssima nota introdutória sobre os mesmos, estes artigos dão-nos a possibilidade de captar a diversidade de quadros teóricos e de conceitos emergentes que se integram nestes novos referenciais (Hymel \& Swearer, 2015). Dos estudos descritivos, permeados de preocupações epidemiológicas, despontam agora os interesses pelas teorias explicativas dos comportamentos de bullying na perspetiva de agressores, de vítimas, e dos que assistem e ocupam papéis diferentes consoante o seu grau de envolvimento nas situações de vitimação (Salmivalli, Lagerspetz, Björkqvist, Österman, \& Kaukiainen, 1996; Swearer \& 
Espelage, 2004; Thornberg, 2015). Realce-se a amplitude sistémica destes novos quadros conceptuais, bem como a necessidade de teorizar os comportamentos de bullying nos contextos, em trajetórias, e em culturas impregnadas de valores e representações que matizam as atitudes e as interações sociais. Merece, a este propósito, dizer que esta tendência evolutiva se reflete em abordagens que reconhecem a necessidade de estudar o bullying, não de maneira isolada, mas de uma forma mais integradora, permitindo compreendê-lo como processo relacional em múltiplos contextos de análise: nas relações de pares, nas amizades, nos grupos identitários e nas relações de pertença, a nível coletivo e societal. Trata-se ainda nestes estudos da importância de considerar modelos de causalidade recíproca para conceptualizar as influências mútuas entre processos de discriminação e de marginalização e os comportamentos de bullying, e como esta cadeia de influências pode potenciá-los ou esvaziá-los de sentido (Carrera, DePalma, \& Lameiras, 2011; Spiel, Salmivalli, \& Smith, 2011).

No primeiro artigo, Melo e Pereira partem do pressuposto de que o bullying é um fenómeno de grupo que tem um impacto apreciável naqueles que assistem e está na base de diferentes estratégias de confronto e diferenças nas atitudes perante os comportamentos de agressores e vítimas. No contexto do grupo, estas diferentes maneiras de lidar refletem-se nos papéis que assumem os seus membros e que variam entre a defesa da vítima ao apoio aos agressores, à adoção de distância e de não envolvimento relativamente a ambos. Outros mecanismos cognitivos e emocionais frequentemente referidos na literatura sobre os comportamentos de bullying estão associados com os motivos e a moralidade (Almeida, Correia \& Marinho, 2010; Caravita, Sijtsema, Rambaran, \& Gini, 2014). No que respeita às disposições para intervir ou não em situações de bullying, será que a empatia, o sentido de auto-eficácia e o descompromisso moral estão relacionadas com os papéis daqueles que assistem (i.e. os observadores)? 0 artigo de Melo e Pereira dá um bom contributo a esta questão através do desenho de uma escala original que avalia os mecanismos motivacionais mostrando que a sensibilidade e a empatia são correlatos de atitudes mais protetoras das vítimas. Inversamente, as atitudes de apoio aos agressores estão correlacionadas com uma maior insensibilidade para reconhecer os efeitos prejudiciais do bullying, uma maior utilização de mecanismos de descompromisso moral (i.e. desengajamento moral) nas situações de bullying e uma menor capacidade de empatia.

0 papel que os pares podem efetivamente ter na resposta de contenção aos comportamentos de bullying num contexto de grupo é, de novo, o foco no segundo artigo de António, Guerra e Moleiro. Estas autoras questionam os fatores que predizem a atuação efetiva dos pares, testando a influência da amizade na prevenção das situações de bullying. Assumindo 
a amizade como um indicador de contacto acrescido, maior empatia e menos perceção de ameaça à imagem de masculinidade ou feminilidade, a sua função protetora reveste-se de maior importância nas situações de bullying homofóbico (Ioverno, Baiocco, Nardelli, Orfano, \& Lingiardi, 2016).

Outra questão de investigação importante diz respeito ao bullying associado a grupos minoritários (e.g. etnia, raça, orientação sexual, género, religião, incapacidade). A dissociação típica dos primeiros estudos de bullying entre pares (Smith, Morita, Junger-Tas, Olweus, Catalano, \& Slee, 1999), que ocultava a relação entre o estatuto identitário dos que se percebiam envolvidos em situações de bullying e o próprio fenómeno, é resolvida e ganha visibilidade nos estudos em que as identidades individuais têm cor e marcador social. Os estudos ancorados teoricamente no quadro da Psicologia Social trazem para os estudos de bullying os referenciais do preconceito, do estereótipo e explicam a formação das atitudes sociais negativas e os efeitos nefastos da homofobia (Herek, Cogan, \& Gillis, 2002; Herek, Gillis, \& Cogan, 2015). Particularmente, ao longo da adolescência estes efeitos são apreciáveis na construção da identidade e no estabelecimento das relações afetivas e românticas com os pares (Russell \& Fish, 2016). Estas metas desenvolvimentais tornam-se desafios mais complicados para os juvenis, cuja identidade de género ou orientação sexual minoritárias, pode ser socialmente estigmatizada. 0 estudo de António, Guerra e Moleiro tem como pano de fundo estas questões e, adicionalmente, propõem-nos que consideremos as 'forças' mas ainda, os riscos que advêm para quem pode assumir uma função social de defesa das vítimas de bullying homofóbico. No paradigma elegante a que nos habituou a Psicologia Social, os resultados elucidam que as intenções para atuar em defesa das vítimas são mediadas pelo contacto prolongado, acréscimo da empatia e decréscimo da ameaça à masculinidade ou feminilidade. Colocando o foco sobre os processos de grupo (i.e., defesa de elementos ou grupos minoritários) e refletindo sobre a importância que neles assumem as funções psicológicas, particularmente, as dimensões afetivas da empatia e o auto-conceito associado ao género e à orientação sexual, as conclusões estabelecem metas muito claras para a intervenção.

O terceiro artigo de Freitas, Coimbra, Fontaine e Marturano assume igualmente um enfoque de tipo sistémico num estudo sobre o ajustamento psicológico em situações de violência social. No pressuposto de que as trajetórias individuais são a expressão de interações complexas e recíprocas de caraterísticas individuais e contextuais (Sameroff, 2009), as autoras consideram que as diferentes formas de violência social tipicamente incluídas nas múltiplas situações de vitimização diretas (agressões físicas, verbais, destruição da propriedade) e indiretas (agressões relacionais, exclusão social e discriminação) têm impactos diferenciados de jovens negros e LGB por comparação com jovens brancos e heterosexuais. Para além de 
referirem que a exposição a situações adversas depende da natureza específica das circunstâncias adversas, acrescentam que, a influência destes fatores pode ser moderada ou mediada pela ação simultânea e conjunta de outros mecanismos individuais ou contextuais, que, exercendo um efeito de proteção, atenuam ou bloqueiam o impacto negativo dos primeiros. A vulnerabilidade ou resiliência são expressão destes processos desenvolvimentais complexos e dinâmicos e não podem ser completamente elucidadas em estudos de índole transversal, mas é possível constatar que a resposta à adversidade de adolescentes de minorias étnicas e sexuais (grupo LGB) revela suscetibilidades específicas de cada grupo. Confirmando algumas das evidências empíricas apontadas na investigação, os resultados deste estudo indicam que há que atender a indicadores de (des)ajustamento internalizados e externalizados e, em cada um destes grupos de indicadores, à presença e ausência das manifestações psicopatológicas que lhes estão associadas. Designadamente, vincando a resposta de inoculação ao stresse dos jovens negros em face de níveis mais elevados de tratamento injusto no dia-a-dia por comparação com a suscetibilidade dos jovens LGB (maior desajustamento externo e menor saúde mental face à vitimização social mesmo na presença de mecanismos de coping ativo). Mas independentemente dos estudos salientarem a força das experiências negativas, não deixa de ser importante revelar a importância dos mecanismos de proteção. Sobretudo quando alguns bons resultados parecem depender mais de "forças mágicas" (Masten, 2014) do que da lógica das coisas, como é o caso do efeito do maior número de ataques à propriedade estar associado a maiores níveis de saúde mental nos jovens LGB.

O quarto artigo da autoria de Campos, Marques, Aguiar, Cardoso, Pinto, e Marques trata das respostas sociais ao bullying quando estas são sancionadas por ações coletivas de justiça popular que, por sua vez, se legitimam na ineficiência dos mecanismos formais de controlo social ou na crença da sua falta de eficiência. A perceção social do quadro normativo que superintende às respostas ao bullying nas escolas - respostas extensíveis ao modo como lidamos socialmente com outras condutas desviantes, está na origem de reações individuais e de processos de grupo cujo fim último é o de assegurar a crença no controlo e na coesão social. Não menos importante são ainda os sentimentos de segurança, conforto e de otimismo relativamente ao futuro e à possibilidade de restaurar as normas do grupo e a imagem associada à legitimidade do grupo. Precisamente, as crenças acerca da fragilidade dos mecanismos de controlo social e consequente impunidade dos agressores pode gerar maior permeabilidade à reposição da justiça pelas próprias mãos como meio de agir em defesa do grupo e legitimar as suas ações em benefício da coletividade. Além disso, a atribuição da responsabilidade aos agressores bem como o favorecimento de ações coletivas associa-se a sentimentos de raiva que funcionam como catalisadores de atos de justiça popular. Os 
resultados deste estudo indicam que as atribuições de responsabilidade são o preditor mais consistente do favorecimento ou defesa das atitudes pró-justiça popular, corroboradas pela raiva e perceção da ineficiência dos mecanismos de controlo. Não obstante, as respostas não apoiam o recurso à justiça popular quando se trata de punir o bullying na escola e desaprovam meios de condenação dos agressores que não adotem as normas instituídas ou escapem aos mecanismos formais de controlo social. Do ponto de vista teórico, os autores confirmam que as emoções negativas (i.e., a raiva) e as crenças da falta de eficiência do controlo social conferem mais peso à associação entre a atribuição de responsabilidade e a aprovação da justiça popular. Na linha de estudos prévios os resultados são ainda congruentes com a ideia de que a justiça e a base legal das normas coletivas são importantes mecanismos de bem-estar e essenciais ao clima de escola e às respostas que os estabelecimentos escolares adotam para fazer face às situações de bullying ou na prevenção das mesmas.

O artigo de Rosário, Candeias e Melo trata de um estudo transverso-longitudinal no qual se pretende avaliar a dimensão e a natureza dos comportamentos de bullying (vitimação, agressão e observação), tomando como momentos inicial e final o $3^{\circ}$ ciclo do ensino básico de um estabelecimento escolar da capital da região alentejana. 0 estudo revisita a importância em distinguir as diferentes formas de bullying (direto físico e verbal e indireto) na perspetiva dos vários papéis assumidos pelos participantes (vitimação, agressão e observação). A investigação recorda que numa perspetiva socio-ecológica, os comportamentos de bullying traduzem um problema sistémico e complexo em que importa atender não apenas aos alunos enquanto indivíduos, mas a outros aspetos que ajudem a caracterizar o seu estatuto e a relação com a escola (Swearer, Espelage, \& Napolitano, 2009; Swearer \& Hymel, 2015). Cingindo a amostra às turmas cuja composição se mostrava preservada ao longo do 3 o ciclo e controlado o sentimento de gosto pela escola e o número de amigos na turma e na escola, as autoras propõem-se demonstrar o efeito destas variáveis de natureza sócio afetiva nos comportamentos de bullying em função da idade e do género dos alunos. Os resultados apontam para um efeito moderado do gosto pela escola nos comportamentos de agressão nos alunos que iniciam o $3^{\circ}$ ciclo $7^{0} \stackrel{0}{\text { ano de }}$ escolaridade), revelando a associação entre as condutas agressivas e gostar pouco da escola. Por sua vez, o efeito das amizades na escola e na turma é assinalável nos comportamentos de vitimização. 0 tamanho do efeito associado a ter amigos na escola e na turma é elevado para o 7ํaㅅ ano, reconhecendo a influência protetora do número de amigos na vitimização. Apesar de este efeito diminuir com a evolução da escolaridade, ter amigos na turma continua a ter um efeito moderado de proteção contra a vitimização no 9o ano. Face a estes resultados, as autoras sublinham a congruência que estes conferem ao estatuto atribuído à amizade como uma variável importante na proteção do bullying, sobretudo dos comportamentos de vitimização. 
Além da idade e do género, ambas variáveis com um enorme poder explicativo nos estudos de bullying (Almeida, 1999; Pellegrini \& Bartini, 2000), a rede de amigos constitui um recurso vital para mobilizar forças nos contextos proximais, nomeadamente, ao nível da turma melhorando a qualidade das experiências socias e afetivas.

No sexto e último artigo de Gouveia, Leal e Cardoso, a perspetiva socio ecológica é mais uma vez convocada para quadro teórico-conceptual do programa de prevenção e intervenção em situações de agressão e bullying na escola. A atenção dada ao fenómeno do bullying e à violência na escola convida à reflexão sobre o conceito de bullying e às semelhanças e diferenças entre este e os conceitos de agressão reativa e proativa. Do ponto de vista dos conceitos, as definições apontam para distinções ao nível dos comportamentos (sob a forma direta ou relacional), assim como dos determinantes e mecanismos que operam em vários níveis em interação (Bronfenbrenner, 1979; Bronfenbrenner \& Morris, 1997). Consistente com a investigação, o estudo assume que o comportamento agressivo e o bullying partilham duas funções (Card \& Little, 2006) A agressão reativa fundamenta-se na hipótese da frustraçãoagressão (Berkowitz, 1993); a instrumental é explicada pelo modelo do processamento sociocognitivo que a descreve como um comportamento planeado e controlado por recompensas externas (Crick \& Dodge, 1996; Olweus, 1993; 2010). Adicionalmente, os autores propõem-se investigar a importância do suporte social da família e amigos e o clima de escola e o peso que cada um destes fatores assume na explicação dos comportamentos agressivos. Curiosamente, os resultados do estudo sugerem índices de agressão muito baixos, para os quais é adiantada a hipótese de a visibilidade do fenómeno do bullying ter apoiado ações preventivas e programas de intervenção que se têm demonstrado eficazes na mudança dos padrões de agressão/vitimização (Ttofi \& Farrington, 2011). Não obstante, as autoras verificaram que as agressões reativas, sob a forma direta ou relacional, sobrepõem-se às instrumentais. A par destes resultados, as perceções de suporte social e do clima de escola também são globalmente positivas. Todavia, registou-se um desfasamento entre a satisfação elevada com o suporte disponível e a avaliação em baixa que os jovens fizeram da disponibilidade do apoio social percebido. Quanto aos preditores, seja a forma direta de agressão seja a relacional, os preditores com maior peso explicativo foram o género (fator individual), o suporte social da família, a satisfação com o suporte social percebido e o clima de escola (sem violência, bom ambiente de ensino). Apoiando a tendência que a investigação tem apontado, os rapazes percebem-se mais envolvidos nas diferentes formas do comportamento agressivo. Outras dimensões do clima de escola não deixam de notar a sua importância para a prevenção e resposta às situações de agressão e, consequentemente, de bullying na escola. Neste domínio, a gestão dos recursos e a qualidade das relações interpessoais (professores-alunos e o papel destes como garante das 
normas e de um ambiente propício à aprendizagem) são apontadas aqui como um mecanismo de proteção importante, em particular, para as agressões reativas. Como conclusão deste estudo é ainda apontada a importância do reforço das redes de apoio e de programas que, de forma estratégica, fomentem a inclusão, a pertença e a coesão social e combatam a alineação e o isolamento social.

Em resumo, os artigos neste monográfico constituem uma pequena amostra da investigação sobre bullying em Portugal que nem por isso deixam de refletir a diversidade de questões neste domínio de estudo ou de reivindicar a importância de integrar essa ampla gama de temas num quadro conceptual integrador.

As organizadoras,

Ana Tomás de Almeida (Instituto de Educação- Universidade do Minho) Isabel Correia (ISCTE-IUL)

\section{REFERÊNCIAS}

Almeida, A. (1999). Portugal. In Smith, P., Morita, Y, Junger-Tas, J., Olweus, D., Catalano, R., \& Slee, P. (eds.), The nature of school bullying. A cross-national perspective (174-186). London: Routledge.

Almeida, A., Correia, I., \& Marinho, S. (2010). Moral disengagement, normative beliefs of peer group and attitudes regarding roles in bullying. Journal of School Violence, 9, 23-36. https://doi.org/10.1080/15388220903185639

Berkowitz, L. (1993). Aggression: Its causes, consequences, and control. New York: McGraw-Hill

Bronfenbrenner, U. (1979). The ecology of human development: Experiments by nature and design. Cambridge, MA: Harvard University Press.

Bronfrenbrenner, U. \& Morris, P. (1997). The ecology of developmental process. In W. Damon (Ed.), Handbook of Child Psychology: Theoretical Models of Human development (Vol. I) (pp. 993-1029). New York: Wiley.

Caravita, S., Sijtsema, J., Rambaran, J., \& Gini, G. (2014). Peer influences on moral disengagement in late childhood and early adolescence. Journal of Youth and Adolescence, 43, 193-207. https://doi.org/10.1007/s10964-013-9953-1

Card, N. \& Little, T. (2006). Proactive and reactive aggression in childhood and adolescence: A meta-analysis of differential relations with psychological adjustment. International Journal of Behavioral Development, 30, 466-480. https://doi.org/10.1177/0165025406071904

Carrera, M.V., DePalma, R., \& Lameiras, M. (2011). Toward a more comprehensive understanding of bullying in school settings. Educational Psychology Review, 23, 479-499. https://doi.org/10.1007/s10648-011-9171-x

Crick, N. \& Dodge, K. (1996). Social information-processing mechanisms in reactive and proactive aggression, Child Development, 67, 993-1002. https://doi.org/10.1111/j.14678624.1996.tb01778.x 
Herek, G. M., Cogan, J. C., \& Gillis, J. R. (2002). Victim experiences in hate crimes based on sexual orientation. Journal of Social Issues, 58, 319-339. https://doi.org/10.1111/15404560.00263

Herek, G. M., Gillis, J. R., \& Cogan, J. C. (2015). Internalized stigma among sexual minority adults: Insights from a social psychological perspective. Stigma and Health, 1, 18-34. https://doi.org/10.1037/2376-6972.1.S.18

Hymel, S. \& Swearer, S. M. (2015). Four decades of research on school bullying. American Psychologist, 70, 293-299. https://doi.org/10.1037/a0038928

Ioverno, S., Baiocco, R., Nardelli, N., Orfano, I., \& Lingiardi, V. (2016). Contrasting the homophobic bullying within the Italian schools: the "itgetsbetter@rome" project experience. In S. Russell \& S. Horn (Eds.), Sexual orientation, Gender identity, and schooling: The nexus of research practice and policy. Oxford (UK): Oxford University Press.

Masten, A. (2014). Ordinary magic. Resilience in development. New York: Guilford Press

Olweus, D. (1993). Bullying at school: What we know and what we can do. Cambridge: Blackwell.

Olweus, D. (2010). Understanding and researching bullying. Some critical issues. In S. R. Jimerson, S. M. Swearer, \& D. L. Espelage (Eds.), Handbook of bullying in schools. An international perspective (pp. 9-33). New York: Routledge.

Pellegrini, D. \& Bartini, M. (2000). A longitudinal study of bullying, victimization, and peer affiliation during the transition from primary school to middle school. American Educational Research Journal, 699-725. https://doi.org/10.3102/00028312037003699

Russell, S. T., \& Fish, J. N. (2016). Mental Health in Lesbian, Gay, Bisexual, and Transgender (LGB6T) Youth. Annual Review of Clinical Psychology, 12, 465-487. https://doi.org/10.1146/annurev-clinpsy-021815-093153

Salmivalli, C., Lagerspetz, K., Björkqvist, K., Österman, K., \& Kaukiainen, A. (1996). Bullying as a group process: Participant roles and their relations to social status within the group. Aggressive Behavior, 22, 1-15. https://doi.org/10.1002/(SICI)10982337(1996)22:1<1::AID-AB1>3.0.CO;2-T

Sameroff, A. (2009). The transactional model. In A.J. Sameroff (Ed.), The transactional model of development (pp. 3-21). Washington, DC: American Psychological Association

Smith, P.K., Morita, Y., Junger-Tas, J., Olweus, O., Catalano, R., \& Slee, P. (1999). The nature of school bullying. A cross-national perspective. London: Routledge.

Spiel. C., Salmivalli, C., \& Smith, P. (2011). Translacional research: National strategies for violence prevention in school. International Journal of Behavioral Development, 35, 381382. https://doi.org/10.1177/0165025411407556

Swearer, S. M., \& Espelage, D. L. (2004). Introduction: A social-ecological framework of bullying among youth. In D. L. Espelage \& S. M. Swearer (Eds.), Bullying in American schools: $A$ social-ecological perspective on prevention and intervention (pp. 1-12). Mahwah, NJ: Lawrence Erlbaum Associates.

Swearer, S. M., \& Hymel, S. (2015). Understanding the psychology of bullying: Moving toward a social-ecological diathesis-stress model. American Psychologist, 70, 344-353. https://doi.org/10.1037/a0038929

Swearer, S. M., Espelage, D. L., \& Napolitano, S. A. (2009). Bullying prevention and intervention: Realistic strategies for schools. New York, NY: Guilford Press.

Thornberg, R. (2015). School bullying as a collective action: Stigma processes and identity struggling. Children \& Society, 29, 310 - 320. https://doi.org/10.1111/chso.12058

Ttofi, M. \& Farrington, D. (2011). Effectiveness of school-based programs to reduce bullying: A systematic and meta-analytic review. Journal of Experimental Criminology, 7, 27-56. https://doi.org/10.1007/s11292-010-9109-1 